\title{
Comunicación social, desarrollo comunitario y Plan Nacional para el Buen Vivir. Un acercamiento desde la realidad ecuatoriana
}

\author{
Social communication, community development, \\ and the National Plan for Good Living (Buen Vivir). \\ A approach from an Ecuadorian reality perspective
}

\author{
Martín González González ${ }^{1}$, Justo Luis Pereda Rodríguez ${ }^{2}$ \\ mgonzálezg@ups.edu.ec, justoluisperedarodriguez@gmail.com
}

\begin{abstract}
Resumen
El presente trabajo aborda la comunicación social entendida como comunicación participativa, basada en el diálogo, el intercambio, la voluntad de compartir experiencias y conocimientos, la búsqueda común y el poder de decisión desde las bases, vinculado al desarrollo comunitario, asumido como procesos en virtud de los cuales, la población, con participación desde el poder, une sus esfuerzos junto al apoyo del Estado, sus instituciones y otros actores sociales para mejorar la calidad de vida, a partir del uso racional de sus potencialidades y de las capacidades existentes en su entorno local, tanto económicas, sociales, culturales, naturales como tecnológicas. Las bases del Plan Nacional del Buen Vivir (Constitución de Montecristi, 2008) en la República del Ecuador, se fundamenta en las concepciones antes mencionadas, para el logro de calidad de vida con equidad social, que facilite la diversidad cultural y ambiental, procesos equilibrados, igualdad posible y solidaridad.
\end{abstract}

\section{Palabras claves}

Comunicación, desarrollo comunitario, Buen Vivir.

Forma sugerida de citar:
Gonzáles González, M., \& Pereda Rodríguez, J.L. (2015). Comunicación social, desarrollo comunitario y Plan Nacional para el Buen Vivir. Un acercamiento desde la realidad ecuatoriana. Universitas, XIII (22), pp. 117-137. Quito: Editorial Abya Yala/Universidad Politécnica Salesiana

1 Ph D. Cuba. Profesor Titular. Universidad Politécnica Salesiana de Guayaquil (UPS-G). Ecuador.

2 Ph D. Cuba. Profesor Titular. Universidad Nacional de Educación, Azogues, Cañar. Ecuador. 


\begin{abstract}
This paper addresses the social communication understood as participatory communication, based on dialogue, exchange, the willingness for sharing experiences and knowledge, a common search and decision making power from the basis, linked to community development, assumed as processes in which, the population, with participation from power, it's joining forces together with the support of the State, its institutions and other social actors to improve the quality of life, from the rational use of their potentials and capabilities around their local environment, both economic, social, cultural, natural and technological environment. The principles of the Good Living National Plan (Constitution of Montecristi, 2008) in the Republic of Ecuador, is based on the conceptions mention before, to achieve quality of life with social equity, to facilitate the cultural and environmental diversity, processes balanced, equality and solidarity.
\end{abstract}

\title{
Keywords
}

Communication, community development, Good Living.

\section{Introducción}

La época actual se encuentra regida por la globalización, la creciente mundialización de la economía y los flujos internacionales de la información. Las tecnologías de la información y de la comunicación, junto a otras áreas de la ciencia y la tecnología, son temas de carácter esencialmente internacional y proyectos en expansión, en los planos mundial, regional y local.

Desde esta perspectiva, las profundas transformaciones en el área de la comunicación y la importancia y protagonismo que han adquirido los medios de comunicación en la creación y transmisión de programas, crean nuevas posibilidades de apoyo y colaboración a los proyectos sociales que hoy emprenden sociedades en vías de desarrollo. Esto lleva a acometer la tarea de un diálogo abierto entre diferentes actores sociales que hacen posible el desarrollo, a través de importantes proyectos de desarrollo local.

Cuando en el mundo se imponen las recetas de desarrollo estandarizado, de pensamiento único y elitista, causante de daños irreparables al desarrollo, la educación, la cultura, al medio ambiente, cada vez más los espacios locales y 
comunitarios se reafirman y aceptan, en la dirección de la autogestión y la sustentabilidad, como desarrollo que brinda los recursos necesarios sin dañar al medio ambiente y las posibilidades futuras del hombre. El reconocimiento del desarrollo comunitario, como aspecto importante de la sustentabilidad, se refleja cada vez más en el ámbito de las investigaciones científicas, en documentos importantes.

Esencialmente el trabajo comunitario se fundamenta en identificar una forma de práctica investigativa en la cual grupos de personas, con el apoyo del Gobierno, organizan sus actividades con el objetivo de mejorar sus condiciones de vida y aprender de su propia experiencia, atendiendo a valores y fines compartidos. Su modelo constituye una espiral permanente de reflexión y acción fundamentado en la unidad entre la práctica y el proceso investigativo, el diálogo y la participación con implicación de la población, que se desarrolla a partir de las decisiones del grupo, el compromiso y el avance progresivo. Para lo anterior implementa una estrategia de mejoramiento, observando los efectos de la acción, y se reflexiona colectivamente en torno a los resultados alcanzados, lo que conduce a una nueva planificación y a cambios sucesivos.

En la dirección señalada, el Plan Nacional para el Buen Vivir 2013-2017 es el tercer plan a escala nacional, nutrido de la experiencia de los dos planes anteriores. Por su coherencia y racionalidad, el Plan Nacional para el Buen Vivir pudiera convertirse en un referente para América Latina y el Caribe, aún con imperfecciones, que sobre la marcha, deben y pueden ser corregidas. El éxito de este plan depende de la prioridad que preste el Gobierno y la aceptación de la población, aun cuando existan los obstáculos que presente lo nuevo en su despliegue, y que sirvan de escalones para alcanzar importantes transformaciones en el seno de la sociedad ecuatoriana.

Para su desarrollo en el Plan Nacional, se incluye como línea importante la descentralización como instrumento que dirigirá la implementación de este proceso en el país para los próximos años, atendiendo a consideraciones fundamentales sobre equidad, cohesión territorial, acceso equitativo a los derechos ciudadanos, a la distribución equitativa de los bienes, recursos y servicios públicos, en atención a las condiciones territoriales y sectoriales necesarias para alcanzar el Buen Vivir. El mismo requiere desarrollar una forma de política pública articulada entre planeación, ejecución y evaluación; y propiciar mecanismos de diálogo interactivo y participación ciudadana en las acciones de control y rendición de cuentas, fomentando así la transparencia y la responsabilidad de la gestión pública en todos los niveles de gobierno. Los recorridos del Presiden- 
te, por los diferentes territorios, los resúmenes y comentarios en actos públicos, ratifican esta gestión de gobierno y pueblo. El diálogo abierto y sincero, y la transparencia, serán condiciones del éxito de este Plan Nacional para el Buen Vivir en la República de Ecuador.

El objetivo del presente trabajo consiste en exponer algunos conceptos básicos del proceso de la comunicación social, el desarrollo comunitario y la participación ciudadana, dentro del Programa del Plan Nacional del Buen Vivir en Ecuador.

\section{Desarrollo}

\section{La comunicación social. Un acercamiento}

El término comunicación en una de las más antiguas acepciones derivada de la raíz latina communis, que significa poner en común algo con otro, expresa algo que se comparte, que se sirve o se tiene en común.

Según (González, 2003) históricamente han existido dos formas de entender el término comunicación. Primero como acto de informar, de transmitir, de decir. Segundo, como diálogo, intercambio, relación, de compartir, de corresponder, de reciprocidad. En el desplazamiento de uno a otro, en la actualidad se impone la gran prensa, las televisoras, la radio y otros medos afines, como medios de informar, transmitir, difundir, no pocas veces con una intención manipuladora, desde los centros de poder, el poder mediático, el poder del mercado y de la propia sociedad. Probablemente ahí surgió el inequívoco, la apropiación del concepto comunicación para legitimar privilegios y dominio de los denominados medios de comunicación de masas y esto se ha convertido en paradigma de la comunicación centrada en la transmisión de señales y mensajes.

De este modo, (Kaplún, 1985) en lugar de partir de las relaciones humanas se acudió a la técnica, a la ingeniería y a la electrónica a resguardo de las empresas trasnacionales de los medios donde se informa lo que mejor les aviene y se silencia aquello que compromete las estrategias mediáticas. Las teorías clásicas sobre los medios, basadas en estudios empíricos, señalan que las funciones esenciales de los medios de comunicación son las de informar, formar y entretener. Así se ha entendido desde que comenzó a fraguarse a través de los medios la sociedad de masas en el siglo XIX y sobre todo en el XX y también lo es en 
la actual sociedad- red-global, como la ha llamado el profesor y científico social de nuestro tiempo Manuel Castells.

Los cambios producidos a partir del avance tecnológico entrañan inmensas potencialidades, no obstante también encierran peligros. Nos encontramos ante dos hechos fundamentales: primero, socialmente hemos dado un salto cualitativo sin retorno; segundo, somos parte de una sociedad que está en plena construcción.

Según (Kaplún, 1985) conviene recordar que los medios de comunicación, pese a ser considerados como necesarios para el establecimiento y funcionamiento de las democracias, suelen generar en la práctica una actitud de desconfianza hasta el punto de que los medios sean tildados de "bestias negras" de casi todo. En lo político porque manipulan y descubren realidades que no convienen, enfatiza en lo económico, porque son la base del negocio y del poder de los grupos, y en lo moral porque transmiten productos de baja calidad, son viles, morbosos, sensacionalistas. Pese a estas y otras rigurosas críticas de fondo y forma nadie duda, a estas alturas de la importancia de los medios, hasta el punto de que en los últimos años existe un clamor desde la perspectiva de los científicos sociales y de algunas instituciones públicas para que la educación en materia de comunicación se incorpore a todos los niveles de la enseñanza, comenzando por los más básicos. Muchos países ya han dado este paso adoptando la formación en comunicación como tema transversal en la educación en distintos niveles.

La gravitación que la comunicación social posee en el derrotero cotidiano de la sociedad actual, es determinante; uno de los rasgos salientes del presente planetario es el uso y permanente desarrollo de las tecnologías de la información y la comunicación (TIC) que brindan la posibilidad de una comunicación universal. Esas tecnologías se concentran bajo los designios de los mismos grupos propietarios del poder económico y político, que hegemonizan el control de los medios de comunicación social a nivel mundial. En este sentido, la falta de acceso a la edición de los medios por parte de sectores importantes de la población, permite aseverar que la distribución económica en el manejo de los medios es insuficiente en el proceso de construcción de la subjetividad humana.

Se observa también cada vez mayor relevancia del mercado, lo que genera, como consecuencia la exclusión de algunos sectores sociales, hecho que limita formas de comunicación para la interacción mutua entre estos sectores, principalmente de bajos recursos. No obstante, ellos en sí mismos posibilitan avances antes imposibles de alcanzar. 
Unido a la difusión ideológica, los grandes medios disponen de enormes facilidades para influir sobre las respuestas colectivas de la sociedad. Esta situación de carácter monopólica en materia económica y comunicacional, también se expresa en América Latina, poniendo en tela de juicio la existencia de una verdadera democracia. Estas tendencias subordinan el carácter social de la comunicación al poder económico y avanzan a contramano de una de las conquistas salientes de la comunidad mundial: el derecho a la información y a la libertad de expresión. Es una realidad el poder y las influencias de las redes sociales y la construcción de las generaciones interactivas. La Web es preferida por adolescentes y jóvenes y no pocos adultos de otras edades, un hecho decisivo porque cambió la relación de fuerzas económicas, sociales y culturales de la sociedad digital.

Un hecho también, factible de notar es la incidencia que los medios de comunicación tienen en la sociedad. La cultura y las actitudes comunitarias han recibido el fuerte impacto de los medios masivos y las nuevas tecnologías. La televisión, los multimedios interactivos, las redes satelitales, la internet, han incrementado las posibilidades de información y en consecuencia la relación de las personas con la realidad. Esto representa una situación inédita que atraviesa todos los aspectos de la vida. La comunicación, entendida como sistema social de información, más comunicación y el entretenimiento, se modifican con el empuje tecnológico.

La ensayista inglesa (Hurley, 1998) recuerda que las voces de algunos actores han sido grandemente amplificadas gracias a las nuevas tecnologías. Sectores que "no tenían voz", hoy a la luz de las nuevas tecnologías y lo medios, son reflejados y escuchados. Anteriormente, la capacidad y el derecho para publicar y difundir las opiniones estaban controlados y limitados, y a veces hasta restringidos para uso exclusivo del Estado. En la actualidad, la tecnología permite a cualquier organización o persona publicar o difundir la información a un costo relativamente bajo, de forma rápida y con menos obstáculos, para lograr varios tipos de comunicación: uno a uno, uno a muchos, y muchos a muchos.

En un sentido, la revolución tecnológica está teniendo como consecuencia inmediata que los medios de comunicación resulten cada vez más penetrantes y poderosos. El alcance y la diversidad de los medios de comunicación, accesibles a las personas con paso a los recursos ya son asombrosos: libros y periódicos, televisión y radio, películas y vídeos, grabaciones y comunicaciones electrónicas transmitidas por radio, cable, satélite e Internet. 


\section{Trabajo comunitario y comunicación dialogada}

Los primeros pasos en la creación de una concepción de las ciencias sociales respecto a la participación y comunicación de los grupos, los inicia el psicólogo social alemán radicado en los Estados Unidos (Lewin, 1946).

Se propuso identificar una forma de práctica investigativa en la cual grupos de personas organizan sus actividades con el objetivo de mejorar sus condiciones de vida y aprender de su propia experiencia, atendiendo a valores y fines compartidos. Su modelo constituye una espiral permanente de reflexión y acción fundamentado en la unidad entre la práctica y el proceso investigativo, que se desarrolla a partir de las decisiones del grupo, el compromiso y el avance progresivo. Para lo anterior implementa una estrategia de mejoramiento, observando los efectos de la acción, y se reflexiona colectivamente en torno a los resultados alcanzados, lo que conduce a una nueva planificación y a cambios sucesivos (González M., 2013: 32).

Las primeras experiencias de trabajo comunitario, (Conferencia Nacional) en el sentido técnico moderno, comenzaron en la década del cuarenta, después de la Segunda Guerra Mundial, como programas de trabajo social con el propósito de mejorar las condiciones de vida de algunas comunidades, a partir de sus recursos y el escaso apoyo de los gobiernos en determinadas circunstancias. Como programa, concebía el trabajo comunitario como "el arte de crear, coordinar y sistematizar los agentes instrumentales, a través de los cuales los talentos y recursos de los grupos pueden ser dirigidos hacia la realización de los ideales y potencialidades de sus miembros" (Conferencia Nacional de Servicios Sociales, 1943).

Desde los años 1950, Naciones Unidas y sus organizaciones especializadas comenzaron el uso de la expresión "trabajo comunitario" para expresar aquellos procesos en virtud de los cuales, los esfuerzos de una población se suman a los de su gobierno para mejorar las condiciones económicas, sociales y culturales de la comunidad. Pero no es hasta 1956 que se reúnen la Organización de Naciones Unidas y otras organizaciones internacionales para llegar a acuerdos sobre el trabajo comunitario, concibiéndolo, como el proceso a través del cual los esfuerzos del propio pueblo se unen con el de las autoridades gubernamentales con la finalidad de mejorar las condiciones económicas y culturales de la comunidad, incorporándola a la vida nacional y capacitándola para contribuir al progreso del país. 
Este enfoque, se despliega en la década de los años sesenta, generando una convergencia intelectual de pensamientos y prácticas, hacia los mismos propósitos: promover la acción y la participación popular en base a la comunicación dialógica con el fin de mejorar las condiciones de vida, con carácter emancipatorio, así como una concepción nueva, no tradicional, con programas y proyectos alternativos, de reivindicación, protagonismo y autogestión.

Como una manera intencional de otorgar poder, empowerment, empoderamiento a la gente para que pueda asumir acciones eficaces hacia el mejoramiento de sus condiciones de vida. Es el hecho de llamar a este proceso, investigación, acción, comunicación y conducirlo como una actividad intelectual con una intención emancipatoria (Park, 1990: 37).

En la década de los años 70, y hasta hoy, la concepción de investigación acción y de participación, ha tenido diversas aplicaciones en el desarrollo de los movimientos sociales populares, asociada a la solución parcial de problemas diversos de las comunidades, en el ámbito de la salud, de la cultural, de la historia del pueblo, de la identidad, la teología, con influencia en las masas de creyentes en Latinoamérica y la Filosofía de la Praxis, anunciada y defendida por (Gramsci, 1970) han encontrado lugar en las preocupaciones y debates con el fin de estimular las acciones de las clases desposeídas a partir del conocimiento y potencial humano en el entorno local.

En un sentido amplio, el término comunidad aborda unidades sociales con características que le dan cierta organización dentro de un área determinada. Puede abarcar todos los aspectos de la vida social, un grupo local lo bastante amplio como para contener todas las principales interacciones, todos los status e intereses que componen la sociedad o un grupo cuyos integrantes ocupan un territorio dentro del cual se puede llevar a cabo la totalidad del ciclo de vida. Literalmente, el término comunidad significa cooperación, comunicación, congregación, asociación, relación.

Pensamos que la comunidad puede ser definida:

Como un grupo de personas en permanente cooperación e interacción social, que habitan en un territorio determinado, comparten intereses y objetivos comunes, reproducen cotidianamente su vida, tienen creencias, actitudes, tradiciones, costumbres y hábitos comunes, cultura y valores, que expresan su sentido de pertenencia e identidad al lugar de residencia, poseen recursos propios, un grado determinado de organización económica, política y social, y presentan problemas y contradicciones (Basapollo, 2013: 31). 
Consideramos que la comunidad, por tanto, es una unidad social, constituida por grupos que se sitúan en lo que se pude llamar la base de la organización social, vinculados a los problemas de la vida cotidiana con relación a los intereses y necesidades comunes; alimentación, vivienda, ropa y calzado, trabajo, servicios y tiempo libre, costumbres, tradiciones, hábitos, creencias, cultura y valores; ocupa una zona geográfica con límites más o menos precisos, cuya pluralidad de personas interactúan más entre sí que en otro contexto de la misma índole. De lo anterior se derivan tareas y acciones comunes, que van acompañadas de una conciencia y un sentido de pertenencia muy relacionadas con la historia y la cultura de la comunidad.

A su vez, la comunidad es un organismo social influenciado por la sociedad de la cual forma parte, y a su vez funciona como un sistema, más o menos organizado, integrado por otros sistemas, las familias, los grupos, los líderes formales y no formales, las organizaciones e instituciones, que interactúan, y con sus peculiaridades definen el carácter subjetivo de la comunidad e influyen de una manera u otra en el carácter objetivo, en dependencia de su organización y posición respecto a las condiciones materiales donde transcurren su vida y actividad. Comunidad es un término, un concepto que designa una realidad concreta, las comunidades vivenciales que generalmente pueden clasificarse como comunidades urbanas, rurales y semiurbanos, cada una con características propias.

Las prácticas de políticas internacionales están esencialmente relacionadas con la intervención y el intervencionismo. Ellas están asociadas al poder de unos estados sobre otros, imponiendo cierto comportamiento político, económico, social, tecnológico, cultural o militar. La historia universal está plagada de estas prácticas. Hacia el interior de los países predominan también, en general, las políticas intervencionistas respecto a las relaciones del Estado con las instituciones y de estas, a su vez, con las personas que las integran. A pesar de los convenios internacionales y de los presupuestos teóricos de las ciencias sociales para frenar el intervencionismo, este existe a través de múltiples políticas estatales, sociales y académicas.

Esta concepción interventiva de las prácticas internacionales pasó a las ciencias sociales, a la planificación económica, como formas evidentes y eficaces de intervención económica y social, y de esta a la comunidad, asociada a la práctica de que quien decide es el que tiene los recursos, el poder y la autoridad, ya sea el que administra, el investigador, el experto, desde "arriba", subestimando las energías y potencialidades, el conocimiento y los valores del resto de las personas. 
La intervención como lo opuesto a la participación significa introducción, interposición o intermediación desde una postura de autoridad, de un elemento externo con la intención de modificar, o interferir en el funcionamiento de un sistema o proceso en una dirección dada (cambio direccional, cesación, desarrollo). La intervención produce un proceso de interferencia o influencia. Desde mi punto de vista, la intervención comunitaria se realiza por diferentes vías, medios y propósitos en dependencia de los objetivos propuestos a partir de los intereses de los sujetos. La intervención se justificada cuando incluye aspectos significativos, necesarios a la comunidad, deseados por esta y compatibles a las necesidades, los valores y capacidades potenciales y que de no producirse, no se lograría el cambio. Sólo con una clara idea de un vínculo de trabajo entre equipo y comunidad, y una diferenciación de tareas, son viables los planes de intervención.

Considerada así es una variante de intervención más indirecta, para enfrentar problemas de la comunidad a través del Estado y sus instituciones como medio de apoyarla y fortalecerla con recursos a los cuales no tiene acceso, con el propósito de emprender opciones de empleo, educativas, de salud, servicios a la familia y atención social, entre otros. A pesar de lo necesario de la intervención, en el caso que se justifique su uso, en muchos casos es insuficiente, al limitar una de las partes en la que se aborda, la población comunitaria.

El intervencionismo, que se aplicó desde la colonización hasta la actualidad por el dominio de una nación sobre otra, ha tenido consecuencias para los estilos y métodos que han predominado en el trabajo comunitario, expresado de diferentes formas, como el autoritarismo, el extensionismo que suplanta la cultura y los valores del otro.

\section{Paulo Freire, comunicación y extensión}

Un lugar destacado para el trabajo comunitario lo ejerce la propuesta de educación Popular de Paulo Freire, a partir de la década de los 60 del siglo XX, misma que ha devenido una de las escuelas de más influencia para los sectores populares.

Freire fue el primero en la defensa del saber subyugado de las comunidades, frente al desdén elitista de cierta tradición epistemológica, conminó a los oprimidos de nuestra América a crear su propia pedagogía, que apostó por la esperanza y la lucha libertaria (Memorias Congreso Comunidad, 1970: 23). 
Él concibió la educación popular, no sólo en el sentido didáctico de conocimiento, sino en el de aprender por la búsqueda y la investigación como conocimiento vivo, que resulta de la actividad y que se traslada directamente a la acción, un aprendizaje orientado a la misión generosa de formar y educar desde la vida, para guiar, capacitar y conducir las actuales y futuras generaciones.

Con meridiana claridad (Freire, 1988) refirió que en la investigación tradicional también se produce aprendizaje, pero en ella sólo decide el investigador, en tanto los sujetos que participan reciben órdenes y quedan sin posibilidad de participar en la solución de los problemas. Esto fue lo que él caracterizó como un proceso de extensión, antagónico en sí mismo y no de comunicación.

"La extensión" conlleva a acciones que transforman las relaciones sociales en una "cosa", que la niegan como ser de transformación, un depósito que recibe mecánicamente aquello que el hombre "superior" le ordena para ser "moderno". El conocimiento requiere de acción transformadora sobre la realidad y demanda una búsqueda constante. Implica invención y reinvención. En el proceso de aprendizaje sólo aprende verdaderamente aquel que se apropia de lo aprendido, transformándolo en aprehendido con lo que puede por eso mismo reinventarlo: aquel que es capaz de aplicar lo aprendido-aprehendido a las situaciones concretas (Freire, 1988: 28).

Freire estaba alertando, y a la vez enseñando, contra el gran divorcio de las prácticas y enfoques funcionalistas y positivistas que han brindado en más de un siglo la academia y la política, con sus interpretaciones dogmáticas de la realidad, de la participación de las masas, apelando a métodos tradicionales, verticalistas, autoritarios y antidemocráticos, que contradicen una respuesta participativa y liberadora. El citado autor enseña que la capacitación técnica no debe focalizarse sólo en la perspectiva cientificista. Concebía que para cambiar la actitud de las personas de escasos conocimientos técnicos, se necesita conocer su visión del mundo. Lo contrario sería una invasión cultural contraria al diálogo como base para establecer la comunicación y la educación en una relación de búsqueda y de esfuerzos organizativos, de creación de conciencia y poder desde la base en la renovación de la realidad cotidiana.

Pedagogía del oprimido, importante obra (Freire, 1964), deviene hoy un potente antídoto frente a la cultura de la desesperanza, avalada por la fiebre neoliberal. El oprimido, al que se refiere Freire, es el excluido, por lo que señala que la pedagogía del oprimido debe ser elaborada con él y para él, en tan- 
to hombres o pueblos en la lucha permanente de recuperación de la humanidad (Freire, 1988).

El reto está entonces en lograr combinar los grandes reclamos del país con la consolidación de pequeños "cambios" que vamos generando en las miles de pequeñas y "medianas" acciones, que desde la sociedad civil venimos impulsando. Concebimos la acción estratégica de base con cambios sustantivos al nivel macroeconómico, político y jurídico. Ese es el nuevo desafío que enfrentamos: esa es la nueva forma de pensar y hacer política que necesitamos (Núñez, 1998: 29).

Es evidente que las grandes decisiones tomadas en los altos niveles deben estar acompañadas desde el planteamiento, el apoyo y el compromiso de las poblaciones de la localidad donde interactúan, y es en estos espacios de acción donde se pueden materializar gran parte de los esfuerzos cotidianos, desde una construcción participativa y de autogestión, como nos enseñaba el propio Freire, que si las daciones surgían de arriba, del gobierno o de sus instituciones, debía también partir de abajo, de los sectores populares necesitados.

En opinión el autor este proceso necesariamente requiere de la capacitación y educación de la población a nivel local, del acceso al poder, de poder de decisión y de manejo de los recursos. Desde esta perspectiva, cabe la pregunta, ¿para qué servirían los grandes cambios no construye de forma simultánea el entorno específico donde diariamente se desempeña cada persona en el ámbito local? Esto significa un servicio a las grandes mayorías para encarar la solución de sus problemas, no sólo materiales, sino también el rico acervo de conocimientos, cultura, sentimientos y sueños que se generan desde lo popular. Esto es una posición ética y humanista del problema. Mediante la comunicación se pretende transformar la realidad y abrir los caminos hacia una sociedad más interactiva y justa.

Cuando la referencia es la comunicación como diálogo, cabe hacer una distinción importante a la que se refirió Paulo Freire, en uno de sus ensayos, ¿Extensión o comunicación?: La concientización en el medio rural. Esa obra escrita sobre el rol del agrónomo, contiene una profunda visión de la comunicación en relación a la educación de los sujetos.

El texto, de gran vigencia en pleno siglo XXI constituye una aproximación muy completa a las implicaciones de los procesos de extensión y comunicación en las sociedades, especialmente sub-desarrolladas, en las cuales ha existido una fuerte tradición de dependencia frente a las naciones industrializadas, que 
son vistas como paradigmas de desarrollo y tecnología. El asunto radica en la reflexión acerca de cómo una determinada relación basada en la extensión conlleva necesariamente la dependencia y hegemonía del pensamiento hegemónico. En el modelo comunicativo utilizado por Paulo Freire, se da por sentada la existencia de interlocutores, así como una comunicación horizontal entre sujetos que interactúan en el proceso dialógico donde el aprendizaje es mutuo y la acción de comunicación es bidireccional.

En las relaciones humanas del gran dominio, la distancia social existente no permite el diálogo. Éste, por el contrario, se da en áreas abiertas, donde el hombre desarrolla su sentido de participación en la vida común. El diálogo implica la responsabilidad social y política del hombre (Freire, 1988: 49).

En la obra señalada la extensión es lo contrario a la comunicación, pues busca convertir al sujeto, a la sociedad en "cosa" u objeto de los planes de desarrollo, negándole la oportunidad de ser transformador de sus propias relaciones con el mundo. La extensión se contrapone también al conocimiento, puesto que en ella el sujeto recibe dócilmente el contenido que otro le impone, sin permitirle reflexión crítica, de inconformidad, en la búsqueda de una realidad que se investiga por los grupos con el fin de mejorarla, es decir superarla.

Por otra parte, Freire ve en la extensión lo que llama invasión cultural, en donde se niega el diálogo, y se educa tradicionalmente, esclavizando, dominando, negando al sujeto como actor de la historia. Por ello, se debe ligar el trabajo del hombre y la mujer a la concientización del contexto y de la cultura popular. En síntesis, el ser humano debe ser el centro de la discusión.

En Freire (1988) lo que ha de buscar así el extensionista es "extender" sus conocimientos y sus técnicas. Lo que hará Freire, por su parte es un análisis lingüístico del término para definir sus parámetros. Tal análisis conlleva a sentidos como: transmisión por parte de un sujeto activo, contenidos a entregar, mesianismo, superioridad (de quien entrega el contenido), inferioridad (de quien recibe), mecanicismo, e invasión cultural (Freire, 1988: 49).

En el texto (Freire, 1988) la extensión, el extensionista busca que el otro cambie los conocimientos asociados a su acción sobre la realidad (relación hombre-mundo) y sustituya sus formas de enfrentar la naturaleza. El problema es gnoseológico, refiere Freire en su obra, en tanto que las relaciones del hombre-mundo - constitutivas del conocimiento humano- se ven afectadas desde la extensión, puesto que ella tiene implícita la acción de llevar, de transferir, de 
entregar, de depositar algo en decir, resalta dentro de sí una connotación indiscutiblemente mecanicista alguien, es. Es necesaria esta filosofía lo proyectos sociales de transformación local, puesto que los sujetos conocen, tienes experiencias vividas, ideas e iniciativas propias que incluidas en el proceso de diálogo, aportan responsabilidad compartida y sentido de pertenencia. (Freire, 1969).

Freire amplía y precisa sobre el asunto de la comunicación y la extensión al señalar que:

(...) en la dimensión humana, el conocimiento no es el acto a través del cual un sujeto -transformado en objeto- recibe dócil y pasivamente los contenidos que otro le da o le impone. Por el contrario, el conocimiento exige una presencia curiosa del sujeto frente al mundo. De suerte que la extensión limita la capacidad crítica, el mostrar sin revelar, sin descubrir. El hombre es el ser de la praxis, de la acción y la reflexión, en sus relaciones con el mundo es un ser-en-situación, un sujeto responsable de sus propias acciones. La invasión cultural consiste en la penetración que hacen los invasores en el contexto cultural de los invadidos, imponiendo a éstos su visión del mundo, en la medida misma en que frenan su creatividad, inhibiendo su expansión (Freire, 1988: 23).

Es la cultura dominante, extensionista, según el criterio anterior, la que posee los privilegios, mientras que los demás permanecen como simples espectadores, siendo este el panorama ideal para que la extensión se lleve a cabalidad.

Por consiguiente, Freire considera que no es posible comprender el pensamiento sin su doble función cognitiva y comunicativa. Pero esta comunicación no implica una simple extensión de contenidos, es decir, reproducir conocimientos a unos sujetos en una relación vertical de una sola vía, sino por el contrario, exige una estructura dialógica de sujetos activos que intercambian significados en una interrelación. Para que esto se lleve a cabo de forma eficiente es necesario que exista entre los interlocutores unos mínimos de reconocimientos semióticos, semánticos y lingüísticos, estos mínimos permiten una comprensión y por lo tanto una aprehensión de los significados comunicativos, en relación a una visión de la comunicación como construcción participativa de conocimiento desde la libertad y la acción, que permita, el conocimiento de esa realidad e implica la transformación de la misma.

Mediante la comunicación se pretende transformar la realidad y abrir los caminos hacia una sociedad más justa y democrática, señalado en la obra de Freire donde encontramos este proceso dialógico como punto de partida epis- 
temológico en el ejercicio de una pedagogía para la libertad que se construye a través de un vínculo interpersonal entre sujetos sociales históricos.

Las sociedades modernas se esfuerzan por promover vías alternativas de comunicación a alcanzar el derecho de sus pueblos a participar, de protagonistas desde el poder y la democracia como reclamos a la justicia, la igualdad, el derecho a la salud y a la educación y a un medio ambiente sano, a sociedades prósperas. Este es el caso de la actual sociedad ecuatoriana y su proyecto, el Plan Nacional del Buen Vivir, centra sus fundamentos por este proceso y numerosas prácticas ya dan fe de éxito, aun en medio de obstáculos e incertidumbres, plan cuyo éxito depende de pasar de menos oyentes, receptores y ejecutores de mandatos, a hablar y ser escuchados, pasos importantes en la Revolución Ciudadana, donde los sujetos se manifiestan en una comunicación de base, popular, incipientemente democrática y comunitaria.

\section{Plan Nacional para el Buen Vivir. Participación ciudadana}

Debo reconocer mi plena convergencia intelectual con la propuesta del Plan Nacional del Buen Vivir, a través del cual identifico muchas preocupaciones y sensibilidades que me animan luego de décadas: la necesidad de vincular las esferas políticas, económicas, sociales, territoriales y también de sostenibilidad, precisamente con el fin de desarrollar políticas más integradoras, mejor adaptadas a la realidad de la era moderna. (Edgar Morín, Filósofo y sociólogo francés. Mundo Real).

Dentro de las líneas programáticas establecidas en el Programa de Gobierno y traducidas en el Plan Nacional para el Buen Vivir, el autor del presente trabajo se refiere a aquellas que se vinculan directamente a la participación ciudadana y la descentralización, por la especificidad del tema que aborda y lo hace siguiendo la fidelidad al texto.

A partir de 2007 se ejecutó el Plan Nacional de Desarrollo 2007-2010 como propuesta de cambio, que había sido definida en el "Programa de Gobierno" presentado a la ciudanía para las elecciones de 2006. Este proyecto trazado se venía cumpliendo hasta 2008. Después del mandato recibido de la Constitución de Montecristi, el mismo año, se presentó el Plan Nacional para el Buen Vivir 2009-2013. El mismo se cumplió y los logros están a la vista de todo el país. El 17 de febrero de 2013, el pueblo ecuatoriano eligió un programa de gobierno para que sea aplicado -siempre ceñido a la Constitución de Montecristi-, en el 
nuevo periodo de mandato de la Revolución Ciudadana. Ese programa tiene su reflejo inmediato en el Plan Nacional para el Buen Vivir 2013-2017, constituye la guía de gobierno que el país aspira tener y aplicar en los próximos cuatro años (SENPLADES, 2009: 32).

El Sumak Kawsay o Plan Nacional de Buen Vivir fortalece la cohesión social, los valores comunitarios y la participación activa de individuos y colectividades en las decisiones relevantes, para la construcción de su propio destino y felicidad. Se fundamenta en la equidad con respeto a la diversidad, cuya realización plena no puede exceder los límites de los ecosistemas que la han originado. No se trata de volver a un pasado idealizado, sino de encarar los problemas de las sociedades contemporáneas con responsabilidad histórica (Plan Nacional para el Buen Vivir, 2013: 24). Así se identifica este Plan.

El Plan es un conjunto de objetivos que expresan la voluntad de continuar con la transformación histórica del Ecuador. Sus objetivos son: Consolidar el Estado democrático y la construcción del poder popular. Auspiciar la igualdad, la cohesión, la inclusión y la equidad social y territorial, en la diversidad; mejorar la calidad de vida de la población; fortalecer las capacidades y potencialidades de la ciudadanía; construir espacios de encuentro común y fortalecer la identidad nacional, las identidades diversas, la plurinacionalidad y la interculturalidad, ecuatorianos, hombres y mujeres, indígenas, cholos, afroecuatorianos, blancos, mestizos y montubios; consolidar la transformación de la justicia y fortalecer la seguridad integral, en estricto respeto a los derechos humanos; garantizar los derechos de la naturaleza y promover la sostenibilidad territorial y global. Consolidar (Plan Nacional para el Buen Vivir, 2013: 24)

Las rupturas y aportes programáticos que plantea el Plan se encuentran presentes en los siguientes ejes: la equidad, la Revolución Cultural, a revolución educativa; el territorio y la Revolución Urbana, que están identificados con la equidad, la cohesión y el ordenamiento territorial; la Revolución Agraria y la Revolución del Conocimiento.

En este marco se establece el carácter participativo de la planificación como condición para el logro del Buen Vivir (arts. 275, 276, 278 y 279) se señala que: corresponde a todas las personas, colectividades y diversas formas organizativas participar en las fases y los espacios de la gestión pública, así como en la planificación del desarrollo nacional y local y en la ejecución y el control del cumplimiento de planes de desarrollo, que fomenten la participación y el control social, con el reconocimiento de las diversas identidades y la promoción de 
su representación equitativa en todas las fases de la gestión del poder público (Plan Nacional para el Buen Vivir, 2013).

Para ello se establece el SNDPP. Con ello, la participación ciudadana tiene un efecto expansivo en todos los niveles del Estado. En el ámbito local, la participación ciudadana adquiere la forma de consejos ciudadanos de planificación en municipios y prefecturas y de asambleas locales, para realizar ejercicios de diagnóstico y programación participativa. En lo regional, se crearon espacios participativos con los actores territoriales para el diseño de las agendas zonales, y se constituyeron los consejos ciudadanos sectoriales zonales en entidades como la Secretaría Nacional de Planificación y Desarrollo (SENPLADES); en otros territorios se implementaron veedurías y observatorios a las políticas públicas. En lo nacional, la participación ciudadana tiene presencia en las diferentes carteras de Estado a través de los consejos ciudadanos sectoriales. Asimismo, se realizó la primera Asamblea Plurinacional e Intercultural para el Buen Vivir, que aportó en la construcción de este Plan. Por otro lado, se desarrollaron procesos participativos para la elaboración de las agendas de igualdad, y se está trabajando para la creación de los consejos nacionales para la igualdad.

Lo descrito hasta el momento permite afirmar que, en estos años de Revolución Ciudadana, el balance de la participación en los procesos de planificación y en la construcción de política pública ha sido positivo. Estos resultados reafirman el compromiso de profundizar la participación ciudadana y superar las limitaciones del pasado, arraigadas en una cultura ciudadana contemplativa, pasiva y delegativa, al momento de asumir responsabilidades para transformar y provocar los cambios profundos que la patria necesita, para modificar la inserción en el mundo y para cambiar la arquitectura financiera internacional y su institucionalidad, que protege al capital en detrimento del ser humano (Plan Nacional para el Buen Vivir, 2013).

Este Plan tiene un elemento esencialmente participativo. Primero, se ha nutrido de un programa de gobierno que se construyó colectivamente y se basa en el diálogo y propuestas de los ciudadanos para identificar, a modo de diagnóstico, los principales problemáticas sociales desde un espíritu crítico y de perfeccionamiento.

El medio para la participación ciudadana fueron:

- Talleres, mesas temáticas y grupos

- Veedurías y observatorios

- Talleres participativos de prospectiva a nivel zonal 
- Reuniones con delegados de organizaciones sociales nacionales, consejos sectoriales y asambleas locales.

- Seminario internacional con sus inquietudes sobre el Plan

- Maratones radiales y espacios virtuales (Plan Nacional para el Buen Vivir, 2013).

Son enormes los avances en materia de participación ciudadana. Sin embargo, el reto está en cambiar la actitud, todavía pasiva y contemplativa que subsiste en la ciudadanía. Se trata de dejar atrás una cultura ciudadana tutelada por el Estado. Cambiar la cultura ciudadana supone dar contenido y sentido a la participación ciudadana, a fin de que se apropie de los desafíos que implica la implementación de la reforma del Estado, para construir uno que responda a las dinámicas y particularidades propias de cada localidad. Se debe contar con una participación ciudadana que asuma el reto de poner en marcha el cambio de la matriz productiva en cada uno de los territorios; una ciudadanía que anhele y se apasione con la idea de colocar en el mundo no solo materias primas, sino también conocimiento, servicios y bienes hechos en el Ecuador. Desde esta óptica, la participación ciudadana tiene un rol sustantivo en la sostenibilidad de las líneas programáticas establecidas en el Programa de Gobierno y traducidas en el Plan Nacional para el Buen Vivir (Plan Nacional para el Buen Vivir, 2013).

Este salto cualitativo, de ciudadanía objeto de derechos a ciudadanía sujeto de derechos, es una ruptura con las lógicas de poder del mercado. Rompe con la ilusoria dicotomía entre los saberes populares y el conocimiento científico, construcciones creadas para el ejercicio de la dominación, la discriminación, la exclusión y la inacción políticas.

La construcción de una ciudadanía activa, comprometida y deliberante supone profundizar la reforma institucional del Estado para que la participación ciudadana incida en la gestión pública; requiere, además, generar las condiciones y capacidades para promover, sostener y asegurar procesos ciudadanos que promuevan el Buen Vivir, y para institucionalizar espacios de diálogo de saberes para la generación de acciones igualitarias, solidarias, libres, dignas, responsables, en armonía con los ecosistemas y respetuosas de las cosmovisiones que conforman nuestro Estado plurinacional. (Plan Nacional para el Buen Vivir, 2013: 88).

Entre los derechos para mejorar la calidad de vida se incluyen el acceso al agua y a la alimentación (art. 12), a vivir en un ambiente sano (art. 14), a un hábitat seguro y saludable, a una vivienda digna con independencia de la situación 
social y económica (art. 30), al ejercicio del derecho a la seguridad (art. 31) y a la salud (art. 32 y 136). (Plan Nacional para el Buen Vivir, 2013).

En el marco del sistema nacional de competencias, la Constitución estableció competencias por cada nivel de gobierno, así como la obligación de acompañar su transferencia con los recursos necesarios para su ejercicio, hecho que se consagra como un cambio paradigmático en la historia republicana de Ecuador. Es fundamental resaltar que cada gobierno autónomo descentralizado gestiona las competencias que le corresponden en su jurisdicción territorial y en ese marco ejerce facultades de manera obligatoria y articulada al sistema nacional descentralizado de planificación participativa. Por ende, constituyen socios estratégicos para la materialización de la Estrategia Territorial Nacional, debido a que tanto los lineamientos que plantea esta, como las acciones que adopta el gobierno autónomo descentralizado, se efectivizan en el territorio (Plan Nacional para el Buen Vivir, 2013).

La articulación de actores -gobiernos autónomos descentralizados, nivel central desconcentrado y ciudadanía-junto con la generación de socios estratégicos en el territorio, permite la prestación de servicios públicos a la población bajo la perspectiva del Buen Vivir y respondiendo a los principios de unidad, solidaridad, coordinación, corresponsabilidad, subsidiariedad, complementariedad, equidad interterritorial, participación ciudadana y sustentabilidad. Así la reducción de brechas en gran medida depende de la calidad de la prestación de estos servicios públicos por parte de los gobiernos autónomos descentralizados a la ciudadanía; así como de su capacidad para generar ingresos propios y regulares adecuadamente tasas y tarifas. Debe resaltarse que el proceso de descentralización, conjuntamente con el proceso de desconcentración, son los mecanismos más efectivos para acercar los servicios a la ciudadanía, fortalecer mecanismos de control social y generar equilibrio territorial (Plan Nacional para el Buen Vivir, 2013).

\section{Conclusiones}

1. Históricamente el modelo emisor-mensaje-receptor se ha expresado en nuestras sociedades autoritarias, jerarquizadoras y estratificadas, en una relación de subordinación de padres de familias hacia los hijos, de las instituciones educativas a los estudiantes, de las instituciones profesionales a sus trabajadores, de la gran prensa a los lectores, de las te- 
levisoras a los espectadores, de los gobiernos a los gobernados, de las grandes potencias, a los pueblos del tercer mundo. La relevancia de la comunicación consiste en transmitir ideas, conocimientos, sentimientos, habilidades, mediante el empleo de signos y palabras.

2. La comunidad en su desarrollo, como elemento básico de la estructura social, necesita del apoyo del Estado como asunto priorizado en la estrategia de desarrollo social. El marco de la sustentabilidad de las comunidades debe transcurrir en sociedades nuevas donde impere la dimensión humanista, la cooperación y solidaridad del hombre y de la mujer; esto es posible sólo en sociedades cuyo centro de preocupaciones lo ocupe el hombre y la mujer, en convivencia equilibrada con el medio ambiente, y en estas condiciones, necesariamente, existen y existirán contradicciones y problemas.

3. El paradigma del desarrollo comunitario sustentable debe prevalecer como los procesos en virtud de los cuales, la población, con participación desde el poder, une sus esfuerzos junto al apoyo del Estado, de sus instituciones y otros actores sociales para mejorar la calidad de vida, a partir del uso racional de sus potencialidades y de las capacidades existentes en su entorno local, tanto económicas, sociales, culturales, naturales como tecnológicas.

4. La participación ciudadana en la toma de decisiones es entendida como el resultado de un proceso de cambio en la correlación de fuerzas, como una redefinición en las relaciones de poder entre el Estado y la sociedad civil organizada o no, de hecho y de derecho-. Por lo tanto, es fruto de una construcción social colectiva. En este contexto político, la participación ciudadana no puede ser reducida a un mero instrumento de poder, ni a receptora de servicios públicos con calidad y calidez; la participación ciudadana tiene que ser el dinamizador de los procesos creativos, innovadores e identitarios que permitan seguir avanzando en la construcción de una sociedad mejor. 


\section{Bibliografía}

González, M., Cabrera, Albert, J.S. \& López P.J. (2013). En: Basapollo, L., Cabrera Albert, J.S., Vivir bien o muerte. A Cuba la felicitá il PIL. Un Avvicinamento al concepto di comunitá. Roma: Editrice, Srl, EURISPES.

Freire, P. (1970). Pedagogía del oprimido. Montevideo: Editorial Tierra Nueva, 2a edición.

(1988). Extensión o comunicación. La concientización en el medio rural. México: Siglo XXI Editores S.A. Vigésima primera edición.

González, N., Osuna, N.A., Rodríguez, M., \& Barrios, N. (2003). Comunicación de Grupo. La Habana: Revista Caminos, Centro Martin Luter King, Jr.

González, M. \& Pereda J.L. (2013). Desarrollo comunitario y educación popular. En: Rojas, H. \& Rodríguez, A., Lo sociocultural. Un trabajo pendiente (pp. 143182). La Habana: Ciencias Sociales.

Gramsci. A. (1970). Introducción a la Filosofía de la praxis. Primera edición. Compendio de los apuntes que formaban el cuaderno n. 11 de los cuadernos escritos en la cárcel. Nueva colección ibérica. Ediciones Península, Italia.

Kaplún, M. (1985). El comunicador popular. Quito: Editorial Belén.

Memorias de Congreso Comunidad (1998). La Habana.

Núñez, C. (1998). La revolución ética. México: Instituto Mejicano para el Desarrollo Comunitario.

Plan Nacional de desarrollo/Plan Nacional para el Buen Vivir 2013-20-17. ISBN 9789942-07- 448-5 Popular, p. 261, Madrid.

Fecha de recepción: 23/2/2015; fecha de aprobación: 26/5/2015 